\title{
Comparative Study on the Separate Extraction of Interface and Bulk Trap Densities in Indium Gallium Zinc Oxide Thin-Film Transistors Using Capacitance-Voltage and Current-Voltage Characteristics
}

\author{
Dong-Ho Lee ${ }^{1}$, Dae-Hwan Kim ${ }^{1}$, Hwan-Seok Jeong ${ }^{1}$, Seong-Hyun Hwang ${ }^{1}$, Sunhee Lee ${ }^{2}$, Myeong-Ho Kim ${ }^{2}$, \\ Jun Hyung Lim ${ }^{2}$ and Hyuck-In Kwon ${ }^{1, *}$ \\ 1 School of Electrical and Electronics Engineering, Chung-Ang University, Seoul 06974, Korea; \\ dongho_12@naver.com (D.-H.L.); pccdhkim@naver.com (D.-H.K.); hwanseok518@naver.com (H.-S.J.); \\ ajttjdwlsgus@naver.com (S.-H.H.) \\ 2 Department of Display R\&D Center, Samsung Display Co., Ltd., Yongin 17133, Korea; \\ sunhee77.lee@samsung.com (S.L.); myeongho86.kim@samsung.com (M.-H.K.); \\ lanosjh.lim@samsung.com (J.H.L.) \\ * Correspondence: hyuckin@cau.ac.kr; Tel.: +82-2-820-5293
}

Citation: Lee, D.-H.; Kim, D.-H.; Jeong, H.-S.; Hwang, S.-H.; Lee, S.; Kim, M.-H.; Lim, J.H.; Kwon, H.-I. Comparative Study on the Separate Extraction of Interface and Bulk Trap Densities in Indium Gallium Zinc Oxide Thin-Film Transistors Using Capacitance-Voltage and Current-Voltage Characteristics. Coatings 2021, 11, 1135. https:// doi.org/10.3390/coatings11091135

Academic Editor: Aomar Hadjadj

Received: 28 August 2021

Accepted: 16 September 2021

Published: 18 September 2021

Publisher's Note: MDPI stays neutral with regard to jurisdictional claims in published maps and institutional affiliations.

Copyright: (c) 2021 by the authors. Licensee MDPI, Basel, Switzerland. This article is an open access article distributed under the terms and conditions of the Creative Commons Attribution (CC BY) license (https:/ / creativecommons.org/licenses/by/ $4.0 /)$.
Abstract: The interface and bulk trap densities were separately extracted from self-aligned topgate (SA-TG) coplanar indium gallium zinc oxide (IGZO) thin-film transistors (TFTs) using the low-frequency capacitance-voltage $(C-V)$ characteristics and space-charge-limited current (SCLC) under the flat-band condition. In the method based on the $C-V$ curve, the energy distribution of the interface trap density was extracted using the low-frequency $C-V$ characteristics, and that of the bulk trap density was obtained by subtracting the density of interface trap states from the total subgap density of states (DOS) at each energy level. In the SCLC-based method, the energy distribution of the bulk trap density was extracted using the SCLC under the flat-band condition at high drain-to-source voltages, and that of the interface trap density was obtained by subtracting the density of bulk trap components from the total subgap DOS at each energy level. In our experiments, the two characterization techniques provided very similar interface and bulk trap densities and showed that approximately $60 \%$ of the subgap states originate from the $\mathrm{IGZO} / \mathrm{SiO}_{2}$ interface at the conduction band edge in the fabricated IGZO TFTs, although the two characterization techniques are based on different measurement data. The results of this study confirm the validity of the characterization techniques proposed to separately extract the interface and bulk trap densities in IGZO TFTs. Furthermore, these results show that it is important to reduce the density of interface trap states to improve the electrical performance and stability of fabricated SA-TG coplanar IGZO TFTs.

Keywords: IGZO TFT; density of interface trap states; density of bulk trap states; low-frequency $C-V$ characteristics; SCLC

\section{Introduction}

Since the first report in 2004, indium gallium zinc oxide (IGZO) thin-film transistors (TFTs) have attracted significant attention owing to their excellent electrical properties, high uniformity, and easy fabrication processes [1]. Recently, IGZO TFTs have been widely used as the backplanes in active-matrix organic light-emitting diode (AMOLED) displays [2,3]. However, the electrical stability of IGZO TFTs needs to be further improved to broaden their applications. In TFTs fabricated with disordered semiconductors, such as IGZO, it is very important to obtain precise information about the subgap density of states (DOS) because it strongly affects the electrical properties and stabilities of TFTs [4,5]. To date, a number of studies have been conducted to extract the energy distribution of the subgap DOS in IGZO TFTs using various characterization techniques [6-10]. However, most previous studies 
reported the total density of subgap states, which includes the bulk trap states and gate insulator/channel interface trap states. Crucially, it is important to find the exact origin of the subgap states to further improve the electrical properties and stabilities of IGZO TFTs. Only a few groups have reported separate values for the interface and bulk subgap DOSs in IGZO TFTs using low-frequency capacitance-voltage $(C-V)$ characteristics [11] and the space-charge-limited current (SCLC) under the flat-band condition [12]. However, the methods for extracting the trap densities are based on different characterization data, which often yield different results because of differences in the detectability of the different types of subgap states. Hence, it is very important to compare the values of the interface and bulk subgap DOSs obtained using different characterization techniques to confirm the validity of these techniques and the accuracy of the extracted trap densities.

In this study, we compare the energy distributions of the interface and bulk subgap DOSs extracted using low-frequency $C-V$ characteristics and the SCLC under the flat-band condition. Experiments were conducted using self-aligned top-gate (SA-TG) coplanar IGZO TFTs, which are widely used in the backplanes of commercially available AMOLED televisions [13-15]. Our experimental results demonstrate that the interface and bulk subgap DOSs extracted using the two different techniques exhibit very similar values. The results of this study confirm the accuracy of the extracted interface and bulk subgap DOSs and the validity of the two characterization methods for separately extracting these DOSs in IGZO TFTs.

\section{Experimental}

Figure 1 shows a schematic of the cross section of the fabricated SA-TG coplanar IGZO TFTs. First, a 250-nm-thick Mo layer was deposited and patterned to form the bottom gate electrode (i.e., a light shielding layer) on a polyimide substrate. Next, a buffer layer $\left(\mathrm{SiN}_{x} / \mathrm{SiO}_{2}=30 / 200 \mathrm{~nm}\right)$ was deposited using plasma-enhanced chemical vapor deposition (PECVD). A 40-nm-thick IGZO layer (In:Ga:Zn = 1:1:1 at.\%) was deposited onto the buffer layer by radio-frequency (RF) sputtering. Subsequently, a 140-nm-thick $\mathrm{SiO}_{x}$ layer was deposited by PECVD as a gate insulator, followed by the deposition of a gate metal $(\mathrm{Ti} / \mathrm{Mo}=30 / 250 \mathrm{~nm})$. After deposition and patterning of the gate electrode and gate insulator, 300-nm-thick $\mathrm{SiO}_{x}$ and 200-nm-thick $\mathrm{SiN}_{x}$ layers were sequentially deposited as a passivation layer by PECVD and patterned to form via holes. A metal layer $(\mathrm{Ti} / \mathrm{Al} / \mathrm{Ti}=30 / 600 / 60 \mathrm{~nm})$ was deposited and patterned as the source and drain electrodes. Finally, the devices were thermally annealed at $340{ }^{\circ} \mathrm{C}$ to achieve stable and uniform electrical performance. The channel width $(W)$ and length $(L)$ of the device were designed to be 10 and $5 \mu \mathrm{m}$, respectively. The current-voltage $(I-V)$ characteristics of the TFTs were measured in the dark at room temperature using an Agilent 4156C semiconductor parameter analyzer (Agilent Technologies, Santa Clara, CA, USA).

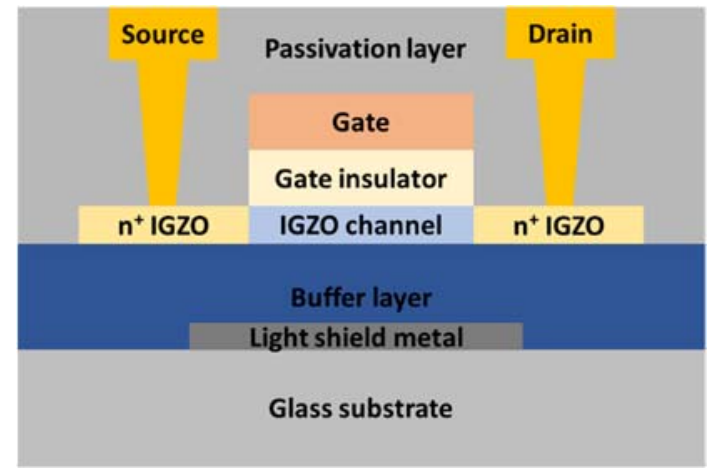

Figure 1. Device structure of the fabricated SA-TG coplanar IGZO TFT.

Figure 2 shows the measurement system for the low-frequency $C-V$ characteristics of the TFTs. A small alternating current (AC) voltage from the function generator (Agilent $33210 \mathrm{~A}$ ) was superimposed on the direct current (DC) voltage provided by a DC power 
supply (Keysight E3646A, Santa Clara, CA, USA), and this voltage signal was applied to the gate electrode of the IGZO TFT located on a probe station. The charging and discharging currents through the source and drain electrodes are expressed as $j \omega C_{\mathrm{GC}} A=A / R_{f}$, where $\omega=2 \pi f, f$ is the frequency of the AC signal, $C_{\mathrm{GC}}$ is the gate-to-source/drain capacitance, $A$ is the amplitude of the AC signal, and $R_{f}$ is the feedback resistance [16]. The signal amplitude and the difference in the phase angles of the sinusoidal current and voltage were confirmed using a digital multimeter (Agilent 34401A, Agilent Technologies Inc., Santa Clara, CA, USA) and lock-in amplifier (EG\&G 5210, EG\&G, Oak Ridge, TN, USA). $C_{\mathrm{GC}}$ can be determined from the obtained value for $A$.

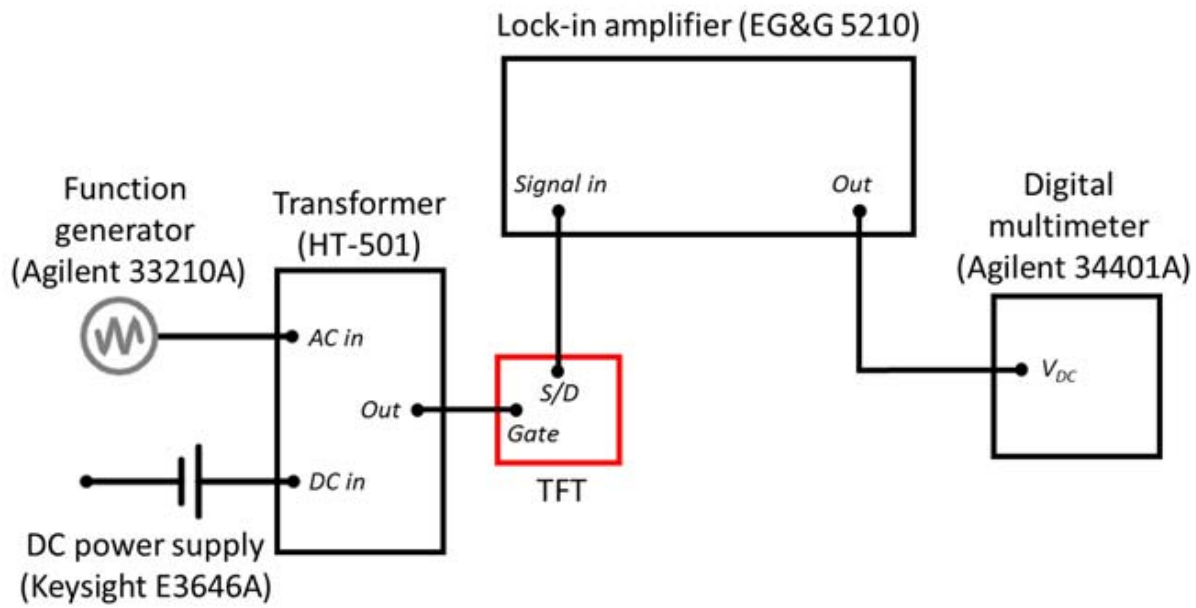

Figure 2. Schematic of the low-frequency $C-V$ measurement system.

\section{Results and Discussion}

Figure 3 shows semilogarithmic and linear-scale transfer curves measured from the fabricated IGZO TFT at a drain-to-source voltage $\left(V_{\mathrm{DS}}\right)$ of $0.1 \mathrm{~V}$. The electrical parameters extracted from a representative device were as follows: a field-effect mobility $\left(\mu_{\mathrm{FE}}\right)$ of $5.9 \mathrm{~cm}^{2} /(\mathrm{V} \cdot \mathrm{s})$, a threshold voltage $\left(V_{\mathrm{TH}}\right)$ of $0.25 \mathrm{~V}$, and a subthreshold swing (SS) of $0.08 \mathrm{~V} /$ dec. Here, $\mu_{\mathrm{FE}}$ was determined using the maximum transconductance method, and $V_{\mathrm{TH}}$ was defined as the gate-to-source voltage $\left(V_{\mathrm{GS}}\right)$ that induces a drain current $\left(I_{\mathrm{D}}\right)$ of $1 \mathrm{nA}$ at $V_{\mathrm{DS}}=0.1 \mathrm{~V}$ [17]. Figure 4 shows the $C-V$ characteristics measured between the gate and source/drain electrodes of the fabricated IGZO TFTs at a low frequency of $103 \mathrm{~Hz}$, where the value of the measurement frequency was chosen to minimize the interference from the power-line harmonics, as per the results of previous reports for a lock-in amplifier [18].

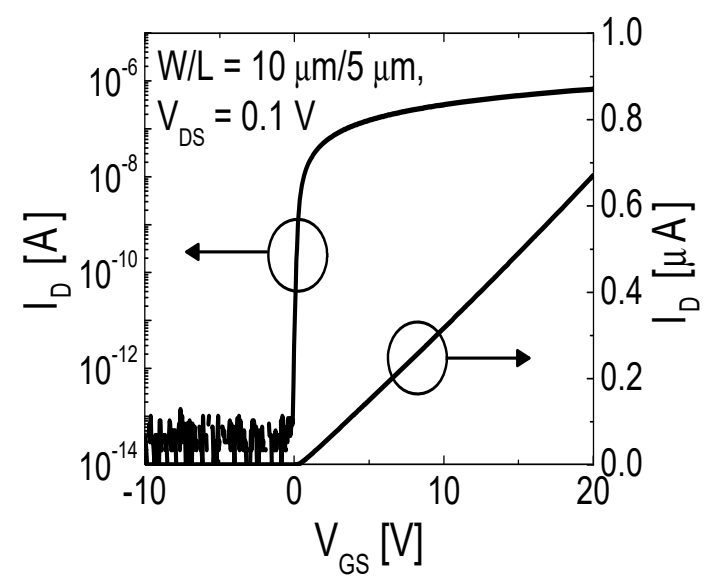

Figure 3. Transfer curve (logarithmic and linear scale) measured from the fabricated IGZO TFT at $V_{\mathrm{DS}}=0.1 \mathrm{~V}$. 


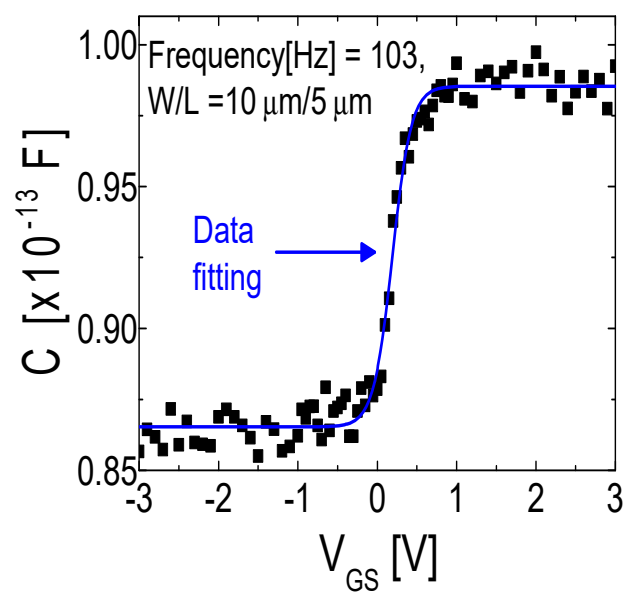

Figure 4. $C-V$ curve measured between gate and source/drain electrodes of the fabricated IGZO TFT at $103 \mathrm{~Hz}$.

In the technique based on the $C-V$ curve, the total density of subgap states is systematically decomposed into interface and bulk trap densities using low-frequency $C-V$ data. The Poisson equation and Gauss's law are numerically solved, and the interface trap density is extracted by fitting the calculated $C-V$ curve to the measured one $[11,19]$. The density of the bulk trap components in the IGZO channel layer is determined by subtracting the density of the interface trap states from the total density of subgap trap states extracted using the transfer curves measured from the TFTs [20]. This technique involves the derivation of two independent equations to solve for the interface trap capacitance $\left(C_{i t}\right)$ and channel capacitance $\left(C_{S}\right)$. The first equation was obtained from the equivalent circuit in Figure $5 \mathrm{a}$, in which $C_{\mathrm{GC}}$ is modeled as

$$
\frac{1}{C_{\mathrm{GC}}}=\frac{1}{C_{\mathrm{OX}}}+\frac{1}{\mathrm{C}_{\mathrm{S}}+\mathrm{C}_{\mathrm{it}}},
$$

where $C_{\mathrm{OX}}$ is the capacitance of the gate insulator $[11,19]$. From Equation (1), $C_{\mathrm{it}}$ can be re-expressed as

$$
C_{\mathrm{it}}=\frac{C_{\mathrm{GC}} C_{\mathrm{OX}}}{C_{\mathrm{OX}}-C_{\mathrm{GC}}}-C_{\mathrm{s}}=\frac{C_{\mathrm{GC}} C_{\mathrm{OX}}}{C_{\mathrm{OX}}-C_{\mathrm{GC}}}-\left(C_{\mathrm{D}}+C_{\mathrm{n}}\right),
$$

where $C_{S}=C_{D}$ (the capacitance of the trapped carriers) $+C_{n}$ (the capacitance of the free carriers) [19]. The second equation for $C_{i t}$ and $C_{S}$ was derived using the Poisson equation and Gauss's law [21]. Figure 5b shows the energy band diagram of the fabricated IGZO TFTs along the channel depth direction. Applying the Poisson equation along the $x$ direction from the channel/gate dielectric interface in Figure 5b, we obtain

$$
\left.\frac{\partial \varphi}{\partial x}\right|_{x=0}=\left[\frac{2 q}{\varepsilon_{0} \varepsilon_{\text {IGZO }}} \int_{0}^{\varphi_{S}}\left(n_{\text {free }}+n_{\text {trap }}\right) d \varphi\right]^{1 / 2},
$$

where $\varepsilon_{0}$ is the vacuum permittivity, $\varepsilon_{\mathrm{IGZO}}$ is the dielectric constant of IGZO, $q$ is the electronic charge, $\varphi$ is the electrostatic potential, and $n_{\text {free }}$ and $n_{\text {trap }}$ are the densities of free and localized trapped electrons, respectively [22].

The trapped charge per unit area $\left(Q_{D}\right)$ and free charge per unit area $\left(Q_{n}\right)$ are expressed by

$$
\begin{aligned}
& Q_{\mathrm{D}}=q \int_{0}^{t_{\mathrm{IGZO}}} n_{\text {trap }} d x=-q \int_{0}^{\varphi_{S}} \frac{n_{\text {trap }}}{d \varphi / d x} d \varphi, \\
& Q_{\mathrm{n}}=q \int_{0}^{t_{\mathrm{IGZO}}} n_{\text {free }} d x=-q \int_{0}^{\varphi_{S}} \frac{n_{\text {free }}}{d \varphi / d x} d \varphi .
\end{aligned}
$$

Adding both sources of charge,

$$
Q_{\mathrm{D}}+Q_{\mathrm{n}}=-q \int_{0}^{\varphi_{S}} \frac{1}{d \varphi / d x}\left(n_{\text {free }}+n_{\text {trap }}\right) d \varphi .
$$


Applying Gauss's law to the channel/gate dielectric interface,

$$
\varepsilon_{0} \varepsilon_{\mathrm{IGZO}} E_{\mathrm{S}}=-\left.\varepsilon_{0} \varepsilon_{\mathrm{IGZO}} \frac{\partial \varphi}{\partial x}\right|_{x=0}=\frac{C_{\mathrm{OX}}\left(V_{\mathrm{GS}}-V_{\mathrm{FB}}-\varphi_{S}\right)}{W L}-Q_{\mathrm{it}},
$$

where $Q_{\mathrm{it}}$ is the interface trap charge per unit area, $V_{\mathrm{FB}}$ is the flat-band voltage, and $E_{\mathrm{S}}$ is the electric field at the IGZO/gate dielectric interface. By substituting Equations (3) and (7) into Equation (6) and differentiating both sides of the equation with respect to $\varphi_{S}$, we obtain the second equation for $C_{\mathrm{it}}$ and $C_{\mathrm{S}}$ as

$$
\begin{aligned}
C_{\mathrm{S}}=C_{\mathrm{D}}+C_{\mathrm{n}} & =W L\left(\frac{d Q_{\mathrm{D}}}{d \varphi_{S}}+\frac{d Q_{\mathrm{n}}}{d \varphi_{S}}\right) \\
& =\frac{1}{2 \eta}\left[C_{\mathrm{Ox}}\left(\frac{d V_{\mathrm{GS}}}{d \varphi_{S}}-1\right)+C_{\mathrm{it}}\right]^{\prime}
\end{aligned}
$$

where $\eta$ is a fitting factor $[11,19]$. The surface potential $\varphi_{\mathrm{S}}$ in Equation (8) can be expressed as a function of $V_{\mathrm{GS}}$ using the transfer characteristics obtained from the TFTs [23]:

$$
\varphi_{\mathrm{S}}=V_{\mathrm{th}} \int_{V_{\mathrm{FB}}}^{V_{\mathrm{GS}}}\left(\frac{\partial \ln I_{\mathrm{D}}\left(V_{\mathrm{GS}}\right)}{\partial V_{\mathrm{GS}}}\right) d V_{\mathrm{GS}}
$$

where $V_{\text {th }}$ is the thermal voltage. Then, we can obtain an equation for $C_{\mathrm{it}}$ by simultaneously solving Equations (2) and (8) [19]:

$$
C_{i t}=\frac{2 \eta-1}{2 \eta+1} \frac{C_{\mathrm{GC}} C_{\mathrm{OX}}}{C_{\mathrm{OX}}-C_{\mathrm{GC}}}
$$

and the interface trap density $\left(D_{\text {it }}\right)$ can be expressed as

$$
D_{\text {it }}=\frac{C_{i t}}{q^{2} W L} .
$$

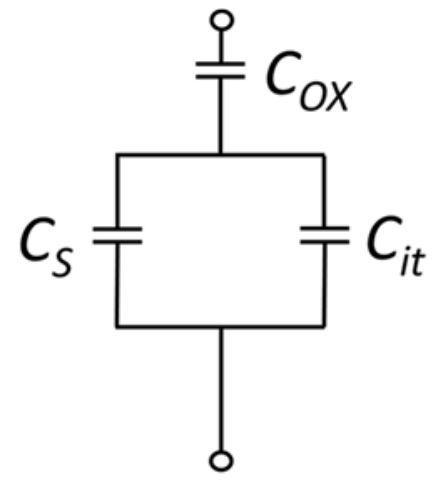

(a)

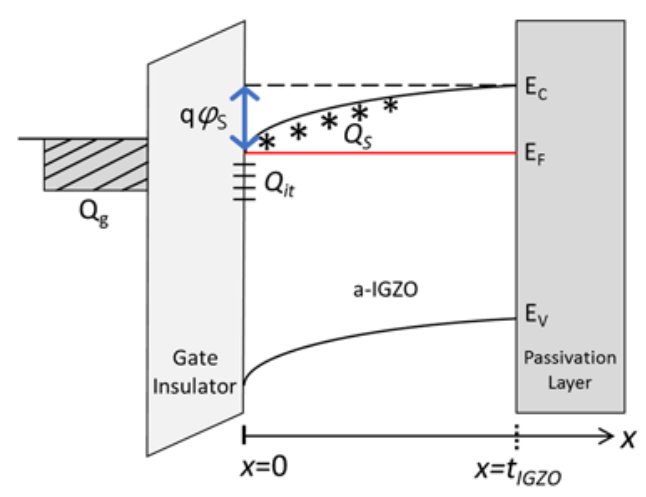

(b)

Figure 5. (a) Equivalent circuit for $C_{\mathrm{GC}}$ with $C_{\mathrm{OX}}, C_{\mathrm{S}}$, and $C_{\mathrm{it}}$; (b) Energy band diagram along the channel depth direction.

By substituting the low-frequency $C-V$ data measured from the TFT in Figure 4 into Equations (10) and (11), the energy distribution of the interface trap states, $D_{\mathrm{it}}(E)$, is calculated as shown in Figure 6a. To determine the density of bulk trap components in the IGZO TFT, we calculated the total density of subgap trap states, which includes the interface and bulk trap states, from the subthreshold characteristics of the IGZO TFT using the following equation and subtracted $D_{\text {it }}$ from it:

$$
\begin{aligned}
D_{\mathrm{it}}+t_{\mathrm{S}} N_{\mathrm{b}} & =\frac{C_{\mathrm{OX}}}{q^{2} W L}\left(\frac{q}{k T \ln 10} S S-1\right) \\
& =\frac{C_{\mathrm{OX}}}{q^{2} W L}\left(\frac{q}{k T}\left(\frac{\partial \ln I_{\mathrm{D}, \text { sub }}}{\partial V_{\mathrm{GS}}}\right)^{-1}-1\right),
\end{aligned}
$$

where $t_{\mathrm{S}}$ is the thickness of the channel layer, $N_{\mathrm{b}}$ is the density of bulk trap states, $k$ is the Boltzmann constant, $T$ is the measurement temperature, and $I_{\mathrm{D} \text {,sub }}$ is the subthreshold drain current $[20,24]$. Figure $6 \mathrm{~b}$ depicts the energy distributions of the total subgap DOS 
$D_{\mathrm{it}}+t_{\mathrm{S}} \times N_{\mathrm{b}}, D_{\mathrm{it}}$, and $t_{\mathrm{S}} \times N_{\mathrm{b}}$ extracted from the fabricated IGZO TFT. Figure $6 \mathrm{~b}$ shows that approximately $57 \%$ of all subgap states are interface trap states at the conduction band edge $\left(E_{C}\right)$ in the fabricated SA-TG coplanar IGZO TFT.
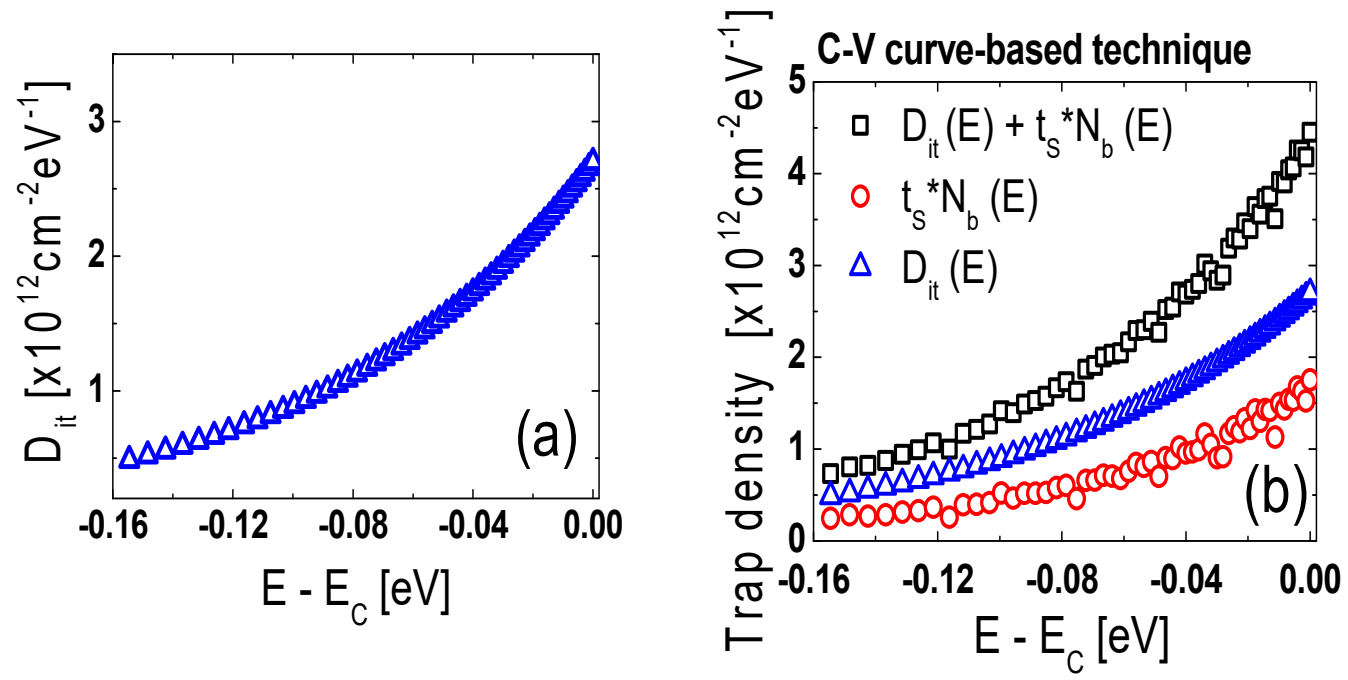

Figure 6. (a) Energy distribution of the density of interface trap states $D_{\text {it }}$ obtained using the technique based on the $C-V$ curve. (b) Energy distributions of the total subgap DOS $D_{i t}+t_{\mathrm{S}} \times N_{\mathrm{b}}$, $D_{\mathrm{it}}$, and the density of bulk trap components $t_{\mathrm{S}} \times N_{\mathrm{b}}$ obtained using the technique based on the low-frequency $C-V$ curve.

In the SCLC-based technique, the total density of subgap states is systematically decomposed into interface and bulk trap densities using SCLC data [12,25]. In this method, we determined the energy distribution of $N_{\mathrm{b}}$ in the IGZO TFT from the SCLC measured under the flat-band condition at a high $V_{\mathrm{DS}}$ [25]. The density of interface traps in the IGZO channel layer was determined by subtracting the density of bulk trap components from the total density of subgap trap states extracted using the transfer curves measured from the TFTs.

Figure 7 shows a log-log plot of the $I_{\mathrm{D}}-V_{\mathrm{DS}}$ curve measured under the flat-band condition after applying the flat-band voltage $\left(V_{\mathrm{FB}}=-0.05 \mathrm{~V}\right)$ to the gate terminal of the TFT. In Figure 7, the measured data can be fitted to the relation $I_{\mathrm{D}} \propto V_{\mathrm{DS}}{ }^{m}$, where $m \sim 1$ at low $V_{\mathrm{DS}}(<5.6 \mathrm{~V})$ and $m \sim 4.04$ at high $V_{\mathrm{DS}}(>5.6 \mathrm{~V})$. The value of $m \sim 1$ at $V_{\mathrm{DS}}<5.6 \mathrm{~V}$ indicates that the $I-V$ characteristics are ohmic at low $V_{\mathrm{DS}}$, and that of 4.04 at $V_{\mathrm{DS}}>5.6 \mathrm{~V}$ indicates that the trap-limited SCLC due to the exponential energy distribution of the trap states within the semiconductor is the dominant current conduction mechanism at high $V_{\text {DS }}$ [26]. An SCLC occurs in semiconductors with low carrier mobilities when the injected charge density exceeds the density of intrinsic free carriers within the semiconductor [27]. From the trap-limited SCLC model, the energy distribution of the density of trap states is exponential and given by

$$
N_{\mathrm{b}}(E)=N_{\mathrm{t}} \exp \left(\frac{E-E_{\mathrm{C}}}{k T_{\mathrm{t}}}\right) \text { where, } T_{\mathrm{t}}=\gamma T \text {. }
$$

The resulting SCLC flowing through the bulk region of the semiconductor between the source and drain terminals under the flat-band condition in the TFT structure is expressed as

$$
I=q W t_{\mathrm{s}} \mu_{\mathrm{n}} N_{\mathrm{c}}\left(\frac{2 \gamma+1}{\gamma+1}\right)^{\gamma+1}\left(\frac{\gamma}{\gamma+1}\right)^{\gamma}\left(\frac{\varepsilon_{0} \varepsilon_{\mathrm{IGZO}}}{q N_{\mathrm{t}} k T_{\mathrm{t}}}\right)^{\gamma} \frac{V^{\gamma+1}}{L^{2 \gamma+1}}
$$

where $N_{\mathrm{t}}$ is the density of bulk trap states at $E_{\mathrm{C}}, T_{\mathrm{t}}$ is the effective temperature of the trap distribution, $\mu_{\mathrm{n}}$ is the bulk carrier mobility, $N_{\mathrm{C}}$ is the effective DOS at $E_{\mathrm{C}}$, and $T_{\mathrm{t}} / T=m-1$ (where $T$ is the measurement temperature). For the fabricated IGZO TFT, $\gamma$ and $k T_{\mathrm{t}}$ were found to be 3.04 and $0.077 \mathrm{eV}$, respectively. By substituting the dimensional parameters 
$\left(W=10 \mu \mathrm{m}, L=5 \mu \mathrm{m}\right.$, and $\left.t_{\mathrm{S}}=40 \mathrm{~nm}\right)$ and electrical parameters $\left(\mu_{\mathrm{n}}=5.9 \mathrm{~cm}^{2} /(\mathrm{Vs})\right.$, $N_{\mathrm{C}}=5 \times 10^{18} \mathrm{~cm}^{-3}, \varepsilon_{\text {IGZO }}=10$, and $\varepsilon_{0}=8.854 \times 10^{-14} \mathrm{~F} / \mathrm{cm}$ ) of the fabricated IGZO TFT into Equations (13) and (14), $N_{\mathrm{b}}(E)$ is calculated as shown in Figure 8a. Figure 8b shows the energy distribution of the total subgap DOS $D_{\mathrm{it}}+t_{\mathrm{S}} \times N_{\mathrm{b}}$, $D_{\mathrm{it}}$, and $t_{\mathrm{S}} \times N_{\mathrm{b}}$ for the fabricated IGZO TFT. Here, $D_{\text {it }}(E)$ was obtained by subtracting $t_{\mathrm{S}} \times N_{\mathrm{b}}(E)$ from the total subgap DOS at each energy level. Similar to the results obtained from the $C-V$ method in Figure $6 \mathrm{~b}$, Figure $8 \mathrm{~b}$ shows that approximately $61 \%$ of the total subgap states are interface trap states at $E_{\mathrm{C}}$ in the fabricated SA-TG coplanar IGZO TFT.

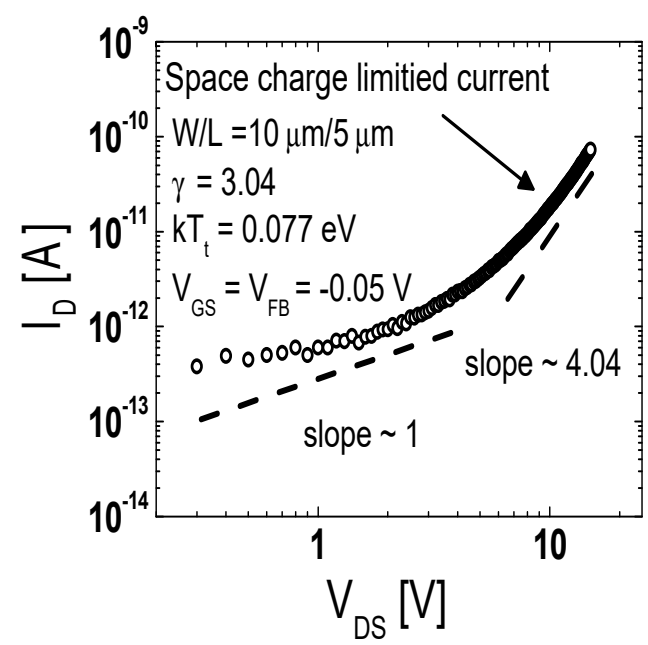

Figure 7. $\log -\log$ plot of the $I_{\mathrm{D}}-V_{\mathrm{DS}}$ curve measured from the fabricated IGZO TFT under the flat-band condition.
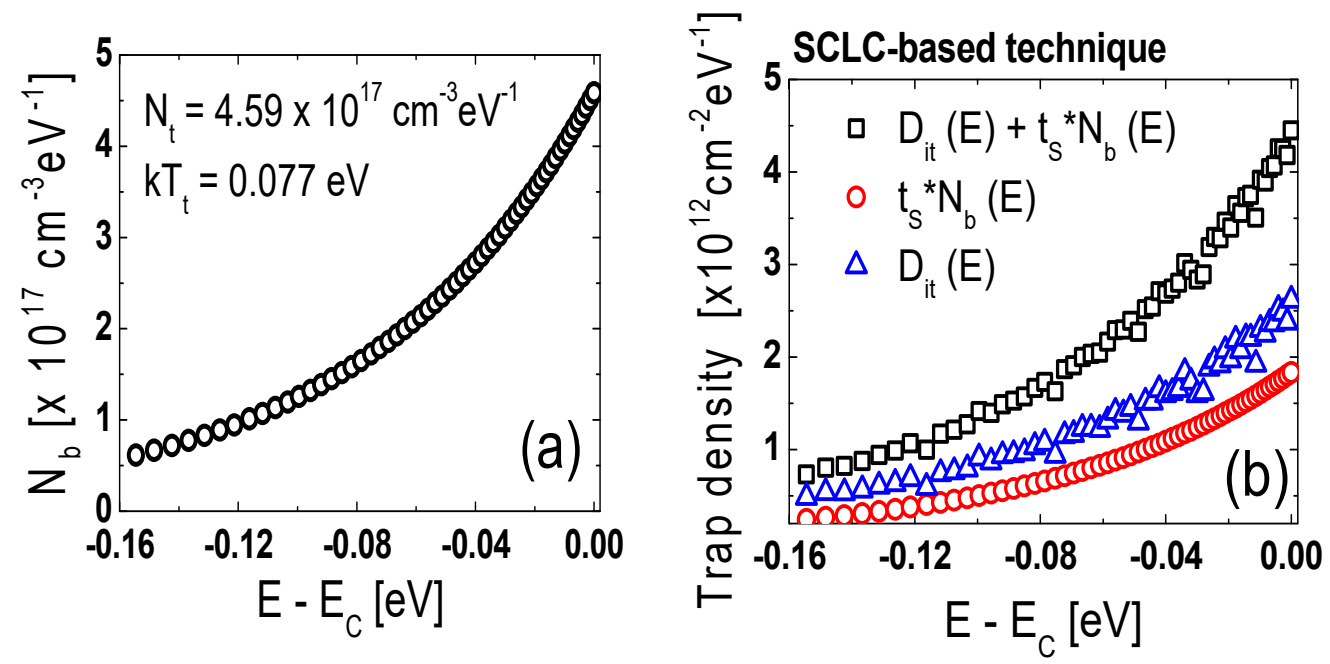

Figure 8. (a) Energy distribution of the density of bulk trap states $N_{\mathrm{b}}$ calculated with the SCLC-based technique. (b) Energy distributions of the total subgap DOS $D_{i t}+t_{\mathrm{S}} \times N_{\mathrm{b}}$, the density of interface trap states $D_{\mathrm{it}}$, and the density of bulk trap components $t_{\mathrm{S}} \times N_{\mathrm{b}}$ obtained using the SCLC-based technique.

Figure $9 \mathrm{a}, \mathrm{b}$ show $D_{\mathrm{it}}(E)$ and $N_{\mathrm{b}}(E)$ for the fabricated IGZO TFT for different extraction methods based on different measurement results. The two characterization techniques provide very similar values of interface and bulk trap densities at every energy level $\left(D_{\text {it }}=2.5 \times 10^{12} \mathrm{~cm}^{-2} \mathrm{eV}^{-1}, N_{\mathrm{b}}=4.1 \times 10^{17} \mathrm{~cm}^{-3} \mathrm{eV}^{-1}\right.$ at $E_{\mathrm{C}}$ for the technique based on the low-frequency $C-V$ curve and $D_{\text {it }}=2.7 \times 10^{12} \mathrm{~cm}^{-2} \mathrm{eV}^{-1}, N_{\mathrm{b}}=4.59 \times 10^{17} \mathrm{~cm}^{-3} \mathrm{eV}^{-1}$ at $E_{\mathrm{C}}$ for the SCLC-based technique), although the two characterization techniques are based on different measurement data. 

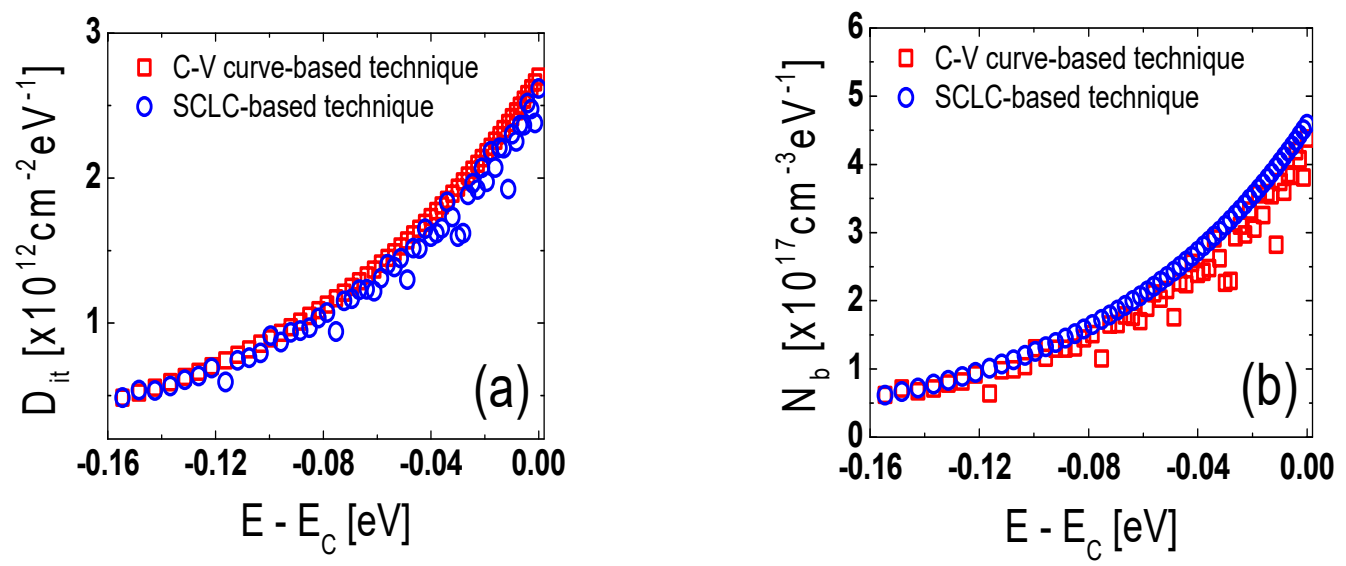

Figure 9. Energy distributions of (a) $D_{\text {it }}$ and (b) $N_{\mathrm{b}}$ extracted using the technique based on the low-frequency $C-V$ curve and the SCLC-based technique.

\section{Conclusions}

In this study, we separately determined the energy distributions of the interface and bulk trap densities from an SA-TG coplanar IGZO TFT using low-frequency $C-V$ characteristics and the SCLC under the flat-band condition. Our experimental results showed that the values of $D_{\text {it }}$ and $N_{\mathrm{b}}$ obtained using the two different techniques exhibited very similar values at every energy level $\left(D_{\mathrm{it}}=2.5 \times 10^{12} \mathrm{~cm}^{-2} \mathrm{eV}^{-1}, N_{\mathrm{b}}=4.1 \times 10^{17} \mathrm{~cm}^{-3} \mathrm{eV}^{-1}\right.$ at $E_{\mathrm{C}}$ for the technique based on the low-frequency $C-V$ curve and $D_{\mathrm{it}}=2.7 \times 10^{12} \mathrm{~cm}^{-2} \mathrm{eV}^{-1}$, $N_{\mathrm{b}}=4.59 \times 10^{17} \mathrm{~cm}^{-3} \mathrm{eV}^{-1}$ at $E_{\mathrm{C}}$ for the SCLC-based technique), although they are based on different measurement data. From both characterization techniques, it may be concluded that $\sim 60 \%$ of the subgap states are attributed to the interface states at $E_{\mathrm{C}}$ in the fabricated SA-TG coplanar IGZO TFT. The results of this study confirm the validity of the characterization techniques for separately extracting the interface and bulk trap densities in IGZO TFTs and show that the density of interface trap states needs to be reduced to improve the electrical properties and stabilities of the fabricated IGZO TFTs.

Author Contributions: Conceptualization, D.-H.L., D.-H.K., S.L., M.-H.K. and H.-I.K.; methodology, D.-H.L., D.-H.K. and H.-S.J.; validation, M.-H.K., J.H.L. and H.-I.K.; investigation, D.-H.L., H.-S.J. and S.-H.H.; data curation, S.-H.H.; writing-original draft preparation, D.-H.L.; writing-review and editing, H.-I.K.; supervision, H.-I.K.; project administration, S.L. and J.H.L. All authors have read and agreed to the published version of the manuscript.

Funding: This research was supported by Samsung Display Co., Ltd. and the National Research Foundation of Korea (NRF) grant funded by the Korea government (MSIT) (2019M3F3A1A03079821, 2020R1A2B5B01001765, 2021M3H2A1038042). This work was also supported by the Industry technology R\&D program (20006400), Next-generation Display Expert Training Project for Innovation Process and Equipment, Materials Engineers (P0012453), and HRD Program for Industrial Innovation (P0017011) funded by the Ministry of Trade, Industry \& Energy (MOTIE) of the Republic of Korea.

Institutional Review Board Statement: Not applicable.

Informed Consent Statement: Not applicable.

Data Availability Statement: Not applicable.

Conflicts of Interest: The authors declare no conflict of interest.

\section{References}

1. Nomura, K.; Ohta, H.; Takagi, A.; Kamiya, T.; Hirano, M.; Hosono, H. Room-temperature fabrication of transparent flexible thin-film transistors using amorphous oxide semiconductors. Nature 2004, 432, 488. [CrossRef] [PubMed]

2. Jeong, J.K.; Jeong, J.H.; Choi, J.H.; Im, J.S.; Kim, S.H.; Yang, H.W.; Kang, K.N.; Kim, K.S.; Ahn, T.K.; Chung, H.; et al. 3.1: Distinguished paper: 12.1-inch WXGA AMOLED display driven by indium-gallium-zinc oxide TFTs. In SID Symposium Digest of Technical Papers; Blackwell Publishing Ltd.: Oxford, UK, 2008; Volume 39, pp. 1-4. 
3. Mo, Y.G.; Kim, M.; Kang, C.K.; Jeong, J.H.; Park, Y.S.; Choi, C.G.; Kim, H.D.; Kim, S.S. Amorphous-oxide TFT backplane for large-sized AMOLED TVs. J. Soc. Inf. Disp. 2011, 19, 16-20. [CrossRef]

4. Yu, E.K.H.; Jun, S.; Kim, D.H.; Kanicki, J. Density of states of amorphous In-Ga-Zn-O from electrical and optical characterization. J. Appl. Phys. 2014, 116, 154505. [CrossRef]

5. De Jamblinne De Meux, A.; Pourtois, G.; Genoe, J.; Heremans, P. Defects in amorphous semiconductors: The case of amorphous indium gallium zinc oxide. Phys. Rev. Appl. 2018, 9, 54039. [CrossRef]

6. Kimura, M.; Nakanishi, T.; Nomura, K.; Kamiya, T.; Hosono, H. Trap densities in amorphous-InGaZn $\mathrm{O}_{4}$ thin-film transistors. Appl. Phys. Lett. 2008, 92,1-4. [CrossRef]

7. Lee, S.; Ahnood, A.; Sambandan, S.; Madan, A.; Nathan, A. Analytical field-effect method for extraction of subgap states in thin-film transistors. IEEE Electron Device Lett. 2012, 33, 1006-1008. [CrossRef]

8. Bae, M.; Yun, D.; Kim, Y.; Kong, D.; Jeong, H.K.; Kim, W.; Kim, J.; Hur, I.; Kim, D.H.; Kim, D.M. Differential ideality factor technique for extraction of subgap density of states in amorphous InGaZnO thin-film transistors. IEEE Electron Device Lett. 2012, 33, 399-401. [CrossRef]

9. Chen, C.; Abe, K.; Kumomi, H.; Kanicki, J. Density of States of a-InGaZnO From Temperature-Dependent Field-Effect Studies. IEEE Trans. Electron Devices 2009, 56, 1177-1183. [CrossRef]

10. Lee, S.; Park, S.; Kim, S.; Jeon, Y.; Jeon, K.; Park, J.H.; Park, J.; Song, I.; Kim, C.J.; Park, Y.; et al. Extraction of subgap density of states in amorphous ingazno thin-film transistors by using multifrequency capacitancevoltage characteristics. IEEE Electron Device Lett. 2010, 31, 231-233.

11. Qiang, L.; Yao, R.H. A new extraction method of trap states in amorphous ingazno thin-film transistors. J. Disp. Technol. 2015, 11, 325-329. [CrossRef]

12. Jeong, C.-Y.; Kim, H.-J.; Kim, J.I.; Lee, J.-H.; Kwon, H.-I. Extraction of bulk and interface trap densities in amorphous InGaZnO thin-film transistors. J. Vac. Sci. Technol. B Nanotechnol. Microelectron. Mater. Process. Meas. Phenom. 2016, 34, 060601. [CrossRef]

13. Geng, D.; Kang, D.H.; Seok, M.J.; Mativenga, M.; Jang, J. High-Speed and Low-Voltage-Driven Shift Register With Self-Aligned Coplanar a -IGZO TFTs. IEEE Electron Device Lett. 2012, 33, 1012-1014. [CrossRef]

14. Song, Z.; Wang, G.; Chen, J.; Gu, P.; Liu, F.; Xie, D.; Liu, W.; Sun, H.; Song, Y.S.; Yan, L.; et al. 24.4: High Performance Top-gate Self-aligned Coplanar a-IGZO TFTs with Light Shielding Metal Design. SID Symp. Dig. Tech. Pap. 2018, 49, 259-262. [CrossRef]

15. Kim, J.B.; Lim, R.; Tsai, Y.C.; Wang, J.; Zhao, L.; Choi, S.Y.; Bender, M.; Yim, D.K. Highly stable self-aligned coplanar InGaZnO thin-film transistors and investigation on effective channel length. Dig. Tech. Pap.-SID Int. Symp. 2019, 50, 874-877. [CrossRef]

16. Sayed, M.B. Solid-State Ac-Conductivity Analysis For The Long Debated Catalytic Activity Of The Boralite Sieve; Qatar University: Doha, Qatar, 1996.

17. Park, J.S.; Jeong, J.K.; Mo, Y.G.; Kim, H.D.; Kim, S. Il Improvements in the device characteristics of amorphous indium gallium zinc oxide thin-film transistors by Ar plasma treatment. Appl. Phys. Lett. 2007, 90, 1-4. [CrossRef]

18. Cultrera, A.; Tran, N.T.M.; D’Elia, V.; Ortolano, M.; Callegaro, L. Calibration of lock-in amplifiers in the low-frequency range. In Proceedings of the 23rd IMEKO TC4 International Symposium Electrical \& Electronic Measurements Promote Industry 4.0, Xi'an, China, 17-20 September 2019; pp. 122-125.

19. Wei, X.; Deng, W.; Fang, J.; Ma, X.; Huang, J. Determination of bulk and interface density of states in metal oxide semiconductor thin-film transistors by using capacitance-voltage characteristics. EPJ Appl. Phys. 2017, 80, 1-5. [CrossRef]

20. Kim, D.H.; Park, M.J.; Kwon, H.I. Separate extraction of densities of interface and bulk trap states in high-mobility ZnON thin-film transistors. J. Nanoelectron. Optoelectron. 2017, 12, 1263-1266. [CrossRef]

21. Bae, M.; Lee, K.M.; Cho, E.S.; Kwon, H.I.; Kim, D.M.; Kim, D.H. Analytical current and capacitance models for amorphous indium-gallium-zinc-oxide thin-film transistors. IEEE Trans. Electron Devices 2013, 60, 3465-3473. [CrossRef]

22. Fung, T.-C. Amorphous In-Ga-Zn-O Thin Film Transistor for Future Optoelectronics. Ph.D. Thesis, University of Michigan, Ann Arbor, MI, USA, 2010.

23. Jun, S.; Jo, C.; Bae, H.; Choi, H.; Kim, D.H.; Kim, D.M. Unified subthreshold coupling factor technique for surface potential and subgap density-of-states in amorphous thin film transistors. IEEE Electron Device Lett. 2013, 34, 641-643. [CrossRef]

24. Fuh, C.S.; Liu, P.T.; Huang, W.H.; Sze, S.M. Effect of annealing on defect elimination for high mobility amorphous indium-zinctin-oxide thin-film transistor. IEEE Electron Device Lett. 2014, 35, 1103-1105. [CrossRef]

25. Kim, H.J.; Kim, D.H.; Jeong, C.Y.; Lee, J.H.; Kwon, H.I. Determination of interface and bulk trap densities in high-mobility p-type $\mathrm{WSe}_{2}$ thin-film transistors. IEEE Electron Device Lett. 2017, 38, 481-484. [CrossRef]

26. Kwan, C.P.; Street, M.; Mahmood, A.; Echtenkamp, W.; Randle, M.; He, K.; Nathawat, J.; Arabchigavkani, N.; Barut, B.; Yin, S.; et al. Space-charge limited conduction in epitaxial chromia films grown on elemental and oxide-based metallic substrates. AIP Adv. 2019, 9, 055018. [CrossRef]

27. Shin, Y.S.; Lee, K.; Kim, Y.R.; Lee, H.; Lee, I.M.; Kang, W.T.; Lee, B.H.; Kim, K.; Heo, J.; Park, S.; et al. Mobility Engineering in Vertical Field Effect Transistors Based on Van der Waals Heterostructures. Adv. Mater. 2018, 30, 1-8. [CrossRef] [PubMed] 